EGU21-10581

https://doi.org/10.5194/egusphere-egu21-10581

EGU General Assembly 2021

(c) Author(s) 2021. This work is distributed under

the Creative Commons Attribution 4.0 License.

\title{
A model for off-fault plastic poroelastic deformation and its effects on permeability
}

Bora Yalcin, Olaf Zielke, and Martin Mai

King Abdullah University of Science and Technology, Physical Science and Engineering, Energy Resources and Petroleum Engineering, Saudi Arabia (bora.yalcin@kaust.edu.sa)

Fractured reservoirs comprise finite or discrete fracture networks; if these are conductive, they form heterogeneously distributed high-permeability streaks. These are generally referred as fracture corridors. Unless they occur as joint swarms, fracture corridors are simply seismic or subseismic fault zones with connected fractures in the near-fault damage zone. Several studies document the decrease in rock-matrix permeability adjacent to the fault surface, within the damage zone. Although the damage zone creates fracture connectivity and high permeability anisotropy for reservoirs, the matrix fracture feeding mechanism is related to matrix permeability generally described by a transfer function. This transfer function accounts for fracture properties (i.e. fracture density, length and connectivity), relative fluid mobilities, imbibition and reservoir properties (i.e. matrix permeability). Commonly, the matrix permeability for all transfer functions is considered in terms of a representative rock type permeability. However, observational evidence and our numerical model show that slip induced deformation causes significant strain on matrix in vicinity to the fault surface causing a permeability decrease in the matrix.

In this study, we present a new approach to model strain in a porous medium and related permeability changes due to stress perturbation from slip around pure strike slip faults. The fault length is used to scale the amount fault slip. For given/computed dislocation (slip) the off-fault strain is then calculated to derive porosity and permeability changes. In our study we propose an off-fault plastic-poroelastic deformation model for any known fault length and known rock mechanical and petrophysical properties of the surrounding material. Our modeling technique will help to better quantify fault transmissivity in geo-reservoirs. 